\title{
MODELOS BAYESIANOS UNIVARIADOS APLICADOS À PREVISÃO DE SÉRIES ECONÔMICAS
}

\author{
Hélio S. Migon*
}

Ana Beatriz S. Monteiro**

Ajax R. B. Moreira***

\section{Resumo}

Modelos de séries temporais são desenvolvidos para diversas séries econômicas brasileiras utilizadas no acompanhamento quantitativo da conjuntura econômica.

A abordagem Bayesiana dos modelos dinâmicos é apresentada, incluindose o desenvolvimento de métodos aproximados de estimação de processos autoregressivos. Discutem-se critérios de monitoramento de mudanças estruturais e apresenta-se uma medida de reversão de tendência da economia.

O desempenho preditivo dos modelos dinâmicos Bayesianos aplicados a um amplo conjunto de indicadores macroeconômicos (indice da produção industrial, balança comercial, componentes do PIB etc.) é apresentado. Esses resultados são, também, comparados com os obtidos a partir de modelos estruturais clássicos e de função de transferência utilizando como regressor dias úteis.

\section{Abstract}

This article reports the results of an exercise in which dynamic models are "fitted" for some Brazilian macroeconomics time series, useful for the current economic analysis. Its forecasting performance is compared with classical structural and transfer function models using trading days as regressor.

The Bayesian forecasting methodology is briefly presented including an alternative way to estimate the parameters of an autoregressive process.

\section{Introdução.}

O acompanhamento da conjuntura econômica pretende interpretar a situação atual e fazer algum tipo de prognóstico para o futuro. Este acompanhamento pode ser realizado, do ponto de vista quantitativo, de uma forma descritiva, observando a tendência de um conjunto

*UFRJ

**UFF

****IPEA

R. de Econometria Rio de Janeiro v. 13, $\mathrm{n}^{\mathrm{Q}} 2$, p.231-259 novembro 1993/abril 1994 
selecionado de séries ou utilizando um modelo formal que relacione estatística e dinamicamente as séries relevantes. Estes procedimentos não se excluem. O uso de um modelo formal explicita as hipóteses do analista e utiliza a interligação entre as séries econômicas para melhor prever, mas pode causar um constrangimento na medida em que limita o analista a um certo corpo de hipóteses quando de fato a explicação dos acontecimentos exige que sejam enfatizados aspectos diferentes a longo do tempo. Neste artigo discute-se apenas os instrumentos estatísticos necessários à abordagem descritiva.

Vale lembrar que a orientação para a previsão de curto prazo torna secundárias questōes muito discutidas atualmente na literatura econométrica como a da existência de raízes unitárias ou da permanência temporal de choques.

O procedimento descritivo utiliza uma estrutura conceitual para identificar as séries relevantes e interpretar suas respectivas tendências. Uma de suas vantagens é a capacidade de acompanhar a volatilidade do fato econômico com a agilidade necessária. Isto envolve o uso da informação com a maior atualidade possível e na mesma periodicidade com que as informações são geradas, a maioria mensalmente. Como as séries frequentemente têm um padrão sazonal bastante significativo torna-se necessário isolar este padrão para extrair a tendência da série.

Os modelos estruturais propostos por Harrison \& Stevens (1976) numa abordagem Bayesiana e por Harvey (1981) numa abordagem clássica postulam que as séries podem ser descritas por componentes como a tendência ou a sazonalidade e substituem outros métodos de decomposição com vantagens, pois tendo fundamento probabilístico são capazes de extrapolar essas componentes e de medir a imprecisão correspondente de forma rigorosa. Modelos estruturais foram utilizados por Migon \& Brasil (1986) e Pereira \& Harvey (1988), respectivamente dentro de uma abordagem Bayesiana e clássica, para a análise da série do produto da indústria geral no Brasil. Neste artigo propõe-se o uso da abordagem Bayesiana para descrever e prever um conjunto de séries mensais e reais relevantes para a análise da conjuntura. As vantagens desta abordagem são ressaltadas através da comparação do desempenho de alguns 
modelos "ajustados" a estas séries, especialmente a do produto da indústria.

Estes modelos admitem componentes estocásticas, permitindo que elas flutuem suavemente ao longo do tempo, o que possibilita o ajustamento local às modificações no processo gerador dos dados. A abordagem Bayesiana dispõe, também, de um procedimento de monitoramento que identifica os períodos em que houve mudanças estruturais, modificações bruscas na trajetória das séries. Nestes períodos o algorítmo é alterado no sentido de torná-lo mais adaptativo às novas condições.

Um tema relevante na literatura econômica é a questão do reconhecimento da reversão da tendência. Supõe-se que a economia tem dois estados, o de recessão e o de crescimento, e a questão consiste em identificar, tão cedo quanto possivel, o momento em que a economia muda de estado. O modelo Bayesiano proposto, além de descrever a série e efetuar previsões será, também, utilizado para derivar uma medida da probabilidade de ocorrência de reversão.

$\mathrm{Na}$ próxima Seção será apresentada a abordagem Bayesiana, incluindo a modelagem de processos autoregressivos de segunda ordem. Critérios de monitoramento para determinação de mudanças estruturais e uma medida de reversão de tendência serão conceituados. $\mathrm{Na}$ Seção 3 modelos Bayesianos, clássicos e de função de transferência para o indice de produção da indústria geral são comparados. O desempenho preditivo do modelo dinâmico Bayesiano para um conjunto razoavelmente amplo de séries: indicadores do produto da indústria, componentes do produto interno bruto, componentes da balança comercial, indicadores de consumo e emprego, é discutido na Seção 4.

\section{Aspectos Metodológicos.}

Os modelos dinâmicos Bayesianos são descritos por um sistema de equações que representam a distribuição das observações e a evolução dos parâmetros. Nesta seção descreve-se as componentes do modelo básico e apresenta-se uma representação para a componente cíclica através de um processo auto-regressivo de segunda ordem. Discute-se um procedimento de identificação de mudanças estruturais na série denominado de monitoramento Bayesiano dos erros 
de previsão e, finalmente, estabelece-se um critério de decisão para a identificação da ocorrência de reversões, momentos em que ocorrem pontos de máximo ou de mínimo na trajetória da série.

\subsection{Modelo Linear Dinâmico Básico.}

Um subconjunto de modelos lineares dinâmicos adequados para representar séries temporais univariadas, incluindo regressores com parâmetros variando, será apresentado a seguir. A série temporal de interesse, $y_{t}$, pode ser representada por duas componentes: nível subjacente $\mu_{t}$ e erro observacional $\epsilon_{t}$, i.é: $y_{t}=\mu_{t}+\epsilon_{t}$.

$O$ nível compreende, em geral, três componentes que aproximam localmente: a tendência, a sazonalidade e efeitos de regressores. A tendência é função polinomial do tempo; a sazonalidade pode ser descrita por fatores sazonais - forma livre - ou através de harmônicos. Nos modelos aditivos essas componentes se superpõe e, então, a série é decomposta em tendência, sazonalidade, efeito dos regressores e erro observacional.

\section{Tendência.}

Uma aproximação local para a tendência envolvendo apenas termos de primeira ordem da expansão de série de Taylor é apresentada abaixo, onde o termo $\mu_{t}^{\prime}$ representa a primeira derivada da tendência e $\epsilon_{t}$ a componente estocástica. Esta aproximação será tão melhor quanto mais suave for a função.

$$
\mu_{t+\delta t} \simeq \mu_{t}+t \mu_{t}^{\prime}+\epsilon_{t}
$$

A função de tendência considerada em momentos equiespaçados no tempo, pode ser representada em cada período pelos parâmetros $\left(\theta_{1}, \theta_{2}\right)$ satisfazendo as equações recursivas abaixo, onde $\omega_{t} \sim$ $N\left[0, W_{t}\right]$. Esses parâmetros correspondem respectivamente ao nível presente $\mu$ e à derivada $\mu^{\prime}$.

$$
\begin{aligned}
& \theta_{1, t}=\theta_{1, t-1}+\theta_{2, t-1}+\omega_{1, t} \\
& \theta_{2, t}=\theta_{2, t-1}+\omega_{2, t}
\end{aligned}
$$

Por substituições sucessivas obtém-se a trajetória esperada da tendência:

$$
\hat{\mu}_{t+k} \simeq \theta_{1, t+k}=\theta_{1, t}+k \theta_{2, t}
$$




\section{Sazonalidade.}

A componente sazonal, de forma análoga, será de natureza estocástica para adaptar-se suavemente às modificações dos dados ao longo do tempo. Se o período sazonal for $s$ podemos descrever a sazonalidade através de $s$ fatores sazonais. As equações abaixo definem, para o caso $s=3$, a dinâmica dos parâmetros, onde $\theta_{1, t}$ é o fator sazonal do mês $t$.

$$
\theta_{1, t}=\theta_{3, t-1}, \quad \theta_{2, t}=\theta_{1, t-1}, \quad \theta_{3, t}=\theta_{2, t-1}
$$

Vale mencionar que funções harmônicas podem substituir os efeitos acima quando se deseja formas restritas e mais parcimoniosas de representar a sazonalidade. Uma ampla discussão desses modelos pode ser encontrada em West \& Harrison (1989), de agora em diante simplesmente WH, Seção 8.4.

\section{Regressores.}

A cada variável independente fica associado um parâmetro que varia suavemente no tempo, o que é útil para considerar omissões de variáveis, mudanças estruturais suaves e representação de relações não lineares através de aproximações lineares locais. $O$ caso de regressão múltipla é obtido pela superposição dos diversos regressores.

\section{Componente Residual.}

A componente residual será representada por uma variável aleatória com distribuição Normal de média zero e variância descrita pela lei do tipo potência: $\operatorname{Var}\left[\epsilon_{t}\right]=c \mu_{t}^{\alpha}$, onde $c$ é um fator de escala e $\alpha$ relaciona a variância residual com a tendência da série. Esta lei de variancia só se aplica, é claro, a séries com tendência não negativa. A constante $c$ será estimada sequencialmente a partir dos dados. A escolha de $\alpha$ pode ser feita em correspondência com a constante envolvida na familia de transformações de Box-Cox.

\subsection{Etapas da Modelagem Dinâmica Bayesiana.}

As principais operações envolvidas na análise Bayesiana são a evolução e atualização. Inicialmente, as distribuições de probabilidade que descrevem os parâmetros do modelo são modificadas de forma a obter-se suas distribuições a priori. Após observar-se uma 
nova realização da série temporal a distribuição a priori é combinada com a verossimilhança gerando a distribuição a posteriori dos parâmetros. A natureza sequencial - define-se para cada período deste método está de acordo com o fato da publicação dos dados e o uso do modelo ocorrerem também de forma sequencial ao longo do tempo.

Dentre as diversas facilidades do método Bayesiano, destacam-se as operações de previsão e suavização, e as possibilidades de tratar observações perdidas e de se fazer intervenções subjetivas.

\section{Modelo Dinâmico Geral.}

As observações, condicionadas aos parâmetros, serão descritas pela seguinte distribuição de probabilidade:

$$
\left(y_{t} \mid \mu_{t}, \phi_{t}\right) \sim N\left[\mu_{t}, \phi_{t}^{-1}\right]
$$

onde: $\phi_{t}=\operatorname{Var}\left(\epsilon_{t}\right)^{-1} \mathrm{e}$

$$
\begin{aligned}
\mu_{t} & =F_{t} \boldsymbol{\theta}_{t} \\
\boldsymbol{\theta}_{t} & =G_{t} \boldsymbol{\theta}_{t-1}+\omega_{t}
\end{aligned}
$$

$\operatorname{com} \omega_{t} \sim N\left[0, W_{t} / \phi_{t}\right]$

$O$ vetor $F_{t}$ e a matriz $G_{t}$ são compostos por blocos que descrevem as diversas componentes. As informações necessárias, no tempo $t-1$, para implementar a análise Bayesiana estão descritas respectivamente pelas distribuições a posteriori normal-gama.

$$
\begin{aligned}
\left(\theta_{t-1} \mid D_{t-1}, \phi_{t-1}\right) & \sim N\left[m_{t-1}, C_{t-1} / \phi_{-1}\right] \\
\left(\phi_{t-1} \mid D_{t-1}\right) & \sim G a\left[n_{t-1} / 2, s_{t-1} / 2\right]
\end{aligned}
$$

onde $D_{t}$ representa a coleção de todas as informações disponíveis até o tempo $t$. Os valores $m_{0}, C_{0}, n_{0} e s_{0}$ que caracterizam as distribuições a posteriori dos parâmetros no instante inicial podem ser determinados subjetivamente ou a partir de prioris de referência como em WH. O próximo passo da análise será descrever a evolução temporal usando (2). 


\section{Evolução e Atualização.}

A expressão (2) descreve, através de $G_{t}$, a evolução determinística do modelo e também o acréscimo de incerteza traduzido pela variância da componente aleatória $\omega_{t}$ permitindo obter-se a distribuição a priori de $\theta$ condicional a $\phi$. A distribuição a priori marginal, $\left(\theta_{t} \mid D_{t-1}\right)$, é obtida integrando-se sobre $\phi$ e é uma t-Student.

Após observar-se $y_{t}$ combinamos a distribuição a priori conjunta de $\left(\theta_{t}, \phi_{t}\right)$ com a verossimilhança para se obter a distribuição a posteriori dos parâmetros a qual é, também, uma normal-gama. Estas expressões são obtidas como função do erro de previsão um passo à frente e dos parâmetros da distribuição a priori e podem ser encontradas em WH, Seção 4.5.

\section{Previsão.}

O valor futuro da série é descrito por uma distribuição condicional aos dados passados, denominada distribuição preditiva

$$
\left(y_{t+k} \mid D_{t}\right) \sim T_{n_{t+k}}\left[f_{t+k}, Q_{t+k}\right]
$$

a qual é uma t-Student com $n_{t+k}$ graus de liberdade. Dispondo-se da distribuição conjunta das previsões futuras, $\left(y_{t+1}, \ldots, y_{t+h}\right)$, pode-se obter a distribuição de funções desses valores futuros. Por exemplo, poderíamos estar interessados na soma das previsões da série ao longo dos próximos trimestres, baseada em dados mensais até o último mês observado.

\section{Intervenção e observações perdidas.}

A natureza subjetiva do método Bayesiano permite que se combine informações objetivas com outras de natureza subjetiva. Um exemplo seria a consideração de alterações estruturais esperadas por especialistas para um certo momento futuro. Neste caso a tendência do processo poderia ser acrescida de uma variável aleatória $\delta \sim N\left[m_{\delta}, v_{\delta}\right]$ com parâmetros fornecidos por especialistas, alterando o nível e a variabilidade desta componente. As observações perdidas são consideradas de forma direta, evoluindo o processo - passagem da priori para a posteriori - sem a incorporação de informações novas. Isto equivale a manter o valor esperado dos parâmetros inalterado e a ampliar as incertezas. 


\section{Suavização.}

Os parâmetros do modelo estrutural dinâmico admitem interpretação e variam ao longo do tempo. Isto significa compreender que os aspectos qualitativos dos modelos são mais estáveis do que os quantitativos. Os parâmetros estarão, portanto, aptos a acomodar mudanças estruturais que venham a ocorrer. Prosseguindo com este raciocínio não é difícil conceber a importância da revisão dos parâmetros no instante $t$ após observar os novos dados em $t+k, \quad k>0$. A distribuição dos parâmetros do período $t$ condicionada a dados posteriores a este período será chamada de distribuição suavizada

$$
\left(\theta_{t} \mid D_{T}, \phi_{t}\right) \sim N\left[m^{s}, C^{s} / \phi_{t}\right], \forall t \leq T
$$

As expressões para $m^{s}$ e $C^{s}$ podem ser encontradas em WH.

\subsection{Modelos Autorregressivos de Segunda Ordem.}

Algumas séries econômicas, em princípio, podem ter uma componente que dê conta de um eventual ciclo, não sazonal e estocástico, inerente ao processo gerador da série. Um exemplo seria o nível de atividade econômica que teria subjacente um ciclo de negócios. $\mathrm{Na}$ literatura, autores como Pereira \& Harvey (1988) incorporaram num modelo estrutural esta componente através de um processo autorregressivo de segunda ordem restrito, representado por uma combinação de senos e cosenos que se amortecem no tempo.

No âmbito dos modelos Bayesianos, a inclusão de uma componente deste tipo resultaria num modelo não linear, em que seriam estimados o coeficiente de amortecimento e o período do ciclo. Um modelo $\mathrm{AR}(2)$ irrestrito será escrito na estrutura do modelo dinâmico Bayesiano, após transformações e estimado sequencialmente. Um processo $\mathrm{AR}(2)$ é definido por qualquer uma das expressões abaixo, onde $\epsilon_{t} \sim N\left[0, \sigma^{2}\right]$ são independentes temporalmente e $\left(\phi_{1}, \phi_{2}\right)$, parâmetros que satisfazem certas restrições e podem variar no tempo.

$$
\begin{aligned}
& y_{t}=a_{t} \\
& a_{t}=\phi_{1} a_{t-1}+\phi_{2} a_{t-2}+\epsilon_{t}
\end{aligned}
$$


Alternativamente pode-se escrever:

$$
y_{t}=\left(\phi_{1}+\phi_{2} B\right) y_{t-1}+\epsilon_{t}
$$

onde $B$ é o operador defasagem. O processo será estacionário se as raízes subjacentes aos parâmetros $\phi$ estiverem fora do círculo unitário. Incluindo o nível na equação (3) obtem-se a expressão abaixo.

$$
y_{t}=\mu_{t}+\left(\phi_{1}+\phi_{2} B\right)\left(y_{t-1}-\mu_{t-1}\right)+\epsilon_{t}
$$

Denote por $a_{t-k \mid t-1}=y_{t-k}-\mu_{t-k}$, para $k=1,2$ as quantidades aleatórias com média $\bar{a}_{t-k}=E\left(y_{t-k}-\mu_{t-k} \mid D_{t-1}\right)$. Estas são, respectivamente, a média a posteriori em $t-1$ e a média da distribuição suavizada, a um passo, em $t-2$. Uma aproximação razoável para o modelo em (4) será:

$$
\begin{aligned}
y_{t} & =\mu_{t}+\phi_{1, t} \bar{a}_{t-1}+\phi_{2, t} \bar{a}_{t-2}+\epsilon_{t}, \quad \text { onde } \epsilon_{t} \sim N\left[0, \sigma^{2}\right] \\
\phi_{k, t} & =\phi_{k, t-1}+\omega_{k+1, t}, \quad k=1,2 \\
\mu_{t} & =\mu_{t-1}+\omega_{3, t} \quad \text { e } \omega_{t} \sim N\left[0, W_{t}\right]
\end{aligned}
$$

que é a composição de uma "tendência" e uma regressão nas variáveis $\left(\bar{a}_{t-1}, \bar{a}_{t-2}\right)$.

As principais vantagens dessa representação são, de um lado a maneira irrestrita de considerar o processo $\operatorname{AR}(2)$ e, de outro, a possibilidade da variância residual ser estimada sequencialmente. Formas alternativas de representar componentes ARMA na estrutura dos modelos dinâmicos Bayesianos são encontradas em WH.

A validação empírica destas aproximações pode ser realizada através de simulações (Monte-Carlo) considerando diferentes valores das raízes autoregressivas e da aleatoriedade do modelo. $\mathrm{O}$ exercício apresentado a seguir é, obviamente, apenas uma sugestão da capacidade do modelo discutido estimar os parâmetros autoregressivos num contexto sequencial e bayesiano. A validação efetiva deste procedimento será deixada para um artigo específico. Assim, com o simples intuito de testar a capacidade de estimação do método proposto gerou-se amostras (240 observações) para quatro processos AR(2), com raízes convenientemente escolhidas na região de inversibilidade. 


\section{Comparação entre o Parâmetro Original e o Estimado}

\begin{tabular}{cc|cc}
\hline \multicolumn{2}{c|}{$\phi_{1}$} & \multicolumn{2}{|c}{$\phi_{2}$} \\
\cline { 3 - 4 } Real & Estimada & Real & Estimada \\
0.1 & 0.0919 & 0.9 & 0.8992 \\
1.0 & 1.0202 & 0.89 & 0.9202 \\
0.8 & 0.7645 & 0.16 & 0.1844 \\
-0.9 & -0.8889 & -0.10 & -0.09742 \\
\hline
\end{tabular}

A variância observacional $\left(\sigma^{2}\right)$ foi fixada em 5.0 e estimada sequencialmente obtendo-se para a média de sua distribuição a posteriori variando entre 4.58 e 5.39 , o que corresponde a um erro relativo médio inferior a $5 \%$.

No exemplo 1 , a escolha de $\phi=(.1, .9)$ corresponde a raízes reais próximas da fronteira de inversibilidade; no segundo, as raízes serão complexas conjugadas, pois $\phi=(1, .89)$. O terceiro exemplo, $\phi=(.8, .16)$ envolve uma única raíz real com multiplicidade $2 \mathrm{e}$, finalmente, $\phi=(-.9,-.01)$ fornece raízes no interior do espaço de parâmetros. Como observa-se na tabela anterior, onde são apresentadas as médias a posteriori após a última observação, o procedimento de estimação funciona adequadamente. Além disto, a natureza das raízes não parece dificultar o processo de estimação.

\subsection{Monitoramento dos Erros de Previsão e Mudanças Es- truturais.}

Um problema relevante em séries econômicas é a determinação dos momentos de mudanças estruturais e o consequente uso da intervenção subjetiva. Em modelos dinâmicos Bayesianos dispõe-se de um mecanismo de acompanhamento dos erros de previsão, baseado no fator de Bayes (WH). Mudanças estruturais certamente prejudicam a descrição dos fenômenos em estudo e, eventualmente, comprometem a capacidade de resposta dos modelos. A forma mais simples de se proceder a intervenção consiste em aumentar a variância da evolução dos parâmetros o que corresponde a desprezar mais fortemente as observações passadas. Formas mais elaboradas envolveriam a mo- 
delagem do efeito da mudança estrutural incluindo a quantificação subjetiva desses eventos, como por exemplo, queda no nível de certa magnitude ou mudança de inclinação.

Intuitivamente, ocorrendo no instante $t$ um erro de previsão padronizado superior a duas unidades, é provável que alguma excepcionalidade esteja ocorrendo. Esta pode ser uma observação espúria ou a indicação do início de uma mudança estrutural. Julgar o modelo considerando o erro de previsão acumulado até o instante $t$ torna o procedimento menos sensível do que considerar os erros acumulados localmente.

A densidade preditiva $p\left(y_{t} \mid D_{t-1}, M\right)$ obtida segundo o modelo $M$ serve para avaliar se uma observação futura está de acordo com o modelo especificado. Valores grandes da densidade preditiva indicam a coerência entre o modelo $M$ e os dados. Agregando-se esta função sobre as $s$ últimas observações têm-se:

$$
\begin{aligned}
& p\left(y_{t}, \ldots, y_{t-s} \mid D_{t-s-1}, M\right) \\
& \quad=p\left(y_{t} \mid D_{t-1}\right) p\left(y_{t-1} \mid D_{t-2}\right) \cdots p\left(y_{t-s} \mid D_{t-s-1}\right)
\end{aligned}
$$

a qual é denominada de densidade preditiva conjunta para uma janela de $s$ períodos. Seu valor é interpretado como a verossimilhança do modelo $M$ e presta-se a comparar modelos. A razão entre as verossimilhanças dos modelos $M$ e $M^{\prime}$ é chamado de fator de Bayes de janela $s$ do modelo $M$ com respeito ao modelo $M^{\prime}$ e denotado por $H(t, s)$. Valores grandes deste fator indicam que o modelo $M$ é preferivel ao modelo $M^{\prime}$.

Se o modelo $M^{\prime}$ for semelhante ao $M$ exceto por admitir maior variabilidade dos seus parâmetros, o fator de Bayes pode ser utilizado para identificar a ocorrência de uma mudança estrutural, sinalizada pela preferência pelo modelo $M^{\prime}$. Se $H(t, 1)$ sinalizar a preferência pela modelo $M^{\prime}$ é possível que esteja ocorrendo apenas uma observação espúria. No entanto se $H(t, s)$ para $s>1$ for ainda significativo, então o procedimento está indicando a ocorrência de uma mudança estrutural, pois se está constatando a preferência sistemática pelo modelo $M^{\prime}$.

\subsection{Reversão Cíclica como um Problema de Decisão.}

A probabilidade de ocorrência de uma reversão pode ser calcu- 
lada a partir de uma definição clara de reversão e de um modelo probabilístico para as observações futuras. Utiliza-se a distribuição preditiva e uma função de perda, para representar as consequências de decisões erradas. Inicialmente define-se reversão sobre os dados observados, como em Zellner et al (1987), e a seguir, estenderemos o conceito para a tendência, componente não observável.

Sejam $D_{t}=\left(y_{1}, \ldots, y_{t}\right)$ as informações disponiveis até o período $t$ e considere-se a observação futura $Y_{t+1}$, quantidade incerta gerada pelo mesmo modelo probabilístico que descreve os dados passados. Definiremos uma reversão para baixo, baseada em $\left(Y_{t-1}, Y_{t}, Y_{t+1}\right)$, se $Y_{t-1}<Y_{t}>Y_{t+1}$, a qual será denotada por $R_{b}$. A reversão para cima $R_{c}$ é conceituada de forma análoga. Estas definições se estendem para incluir várias observações passadas e futuras. A tomada de decisão sob incerteza envolve as probabilidade desses eventos, a saber:

$$
\operatorname{Pr}\left[R_{b} \mid D_{t}\right]= \begin{cases}\operatorname{Pr}\left[Y_{t+1}<y_{t} \mid D_{t}\right], & \text { se } y_{t-1}<y_{t} \\ 0 & \text { caso contrário }\end{cases}
$$

As séries econômicas podem ter estruturas mais elaboradas, incluindo componentes sazonais, cíclicas e erro observacional, que pertubam a identificação da trajetória a longo prazo. Por isto, a reversão deve ser caracterizada a partir da tendência ou nível corrente da série. Assim, conceitua-se como a ocorrência de uma reversão para baixo se $\mu_{t-1}<\mu_{t}>\mu_{t+1}$ e não ocorrência se $\mu_{t-1}<\mu_{t}$ ou $\mu_{t}<\mu_{t+1}$. A probabilidade correspondente será:

$$
\begin{aligned}
\operatorname{Pr}\left[R_{b} \mid D_{t}\right] & =\operatorname{Pr}\left[\mu_{t-1}<\mu_{t} \text { e } \mu_{t+1}<\mu_{t} \mid D_{t}\right] \\
& =\operatorname{Pr}\left[\mu_{t-1}<\mu_{t+1}<\mu_{t} \mid D_{t}\right)+\operatorname{Pr}\left(\mu_{t+1}<\mu_{t-1}<\mu_{t} \mid D_{t}\right]
\end{aligned}
$$

Estas probabilidades devem ser calculadas usando-se a distribuição conjunta de $\left(\mu_{t-1}, \mu_{t}, \mu_{t+1} \mid D_{t}\right)$. As parcelas envolvidas nesta expressão são o parâmetro $\mu$ suavizado a um passo, sua posteriori em $t$ e sua priori para $t+1$. Os resultados apresentados baseam-se numa aproximação para aquela probabilidade onde $\mu_{t-1}$ e $\mu_{t}$ são substituídos por seus valores esperados, embora a maneira adequada de calcular-se essas probabilidades seja através de integração numérica. Assim,

$$
\operatorname{Pr}\left[R_{b} \mid D_{t}\right]= \begin{cases}\operatorname{Pr}\left[\mu_{t+1}<m_{t} \mid D_{t}\right], & \text { se } m_{t-1}<m_{t} \\ 0, & \text { caso contrário }\end{cases}
$$


A decisão pela ocorrência ou não de uma reversão envolve a consideração dos custos incorridos numa decisão errada. É necessário incluir uma funcão de perda (L), como por exemplo a descrita na tabela abaixo. onde $a_{1}$ corresponde a identificar a ocorrência de $R_{b}$ e $a_{2}$ a não identificá-la.

\begin{tabular}{ccc}
\hline \multicolumn{3}{c}{ Eventos } \\
\hline Acōes & $R_{b}$ & $\bar{R}_{b}$ \\
\hline$a_{1}$ & 0 & $\mathrm{c}$ \\
$a_{2}$ & $\mathrm{~d}$ & 0 \\
\hline
\end{tabular}

Embora tenhamos salientado o caráter preditivo do problema, nas aplicaçōes nos limitamos a cálculos baseados na posteriori em $\mathrm{t}+1$. Responde-se, de fato, a uma questão mais simples: 'após observar-se o período $t+1$, terá ocorrido em $t$ uma $R_{b}$ ?'.

A perda esperada para cada uma das ações será: $E\left[L\left(a_{1}\right)\right]=$ $c\left\{1 \operatorname{Pr}\left[R_{b}\right]\right\}$ e $E\left[L\left(a_{2}\right)\right]=d \operatorname{Pr}\left[R_{b}\right]$. A ação $a_{1}$ será escolhida se $E\left[L\left(a_{1}\right)\right]<E\left[L\left(a_{2}\right)\right]$ ié. se $\frac{\operatorname{Pr}\left[R_{b}\right]}{1-\operatorname{Pr}\left[R_{b}\right]}<c / d$. Análogamente a identificação de uma reversão para cima é dada por $\frac{\operatorname{Pr}\left[R_{c}\right]}{1-P_{r}\left[R_{c}\right]}<c^{\prime} / d^{\prime}$. A escolha dos custos é subjetiva retratando a gravidade dos alarmes falsos. Assim $c$ muito maior que $d$ indica que identificar um $R_{b}$ em $t$, quando efetivamente não é, é mais custoso do que não considerálo quando de fato ele é um $R_{b}$. Nesta aplicação, por simplicidade utilizamos $c=d$, o que implica em considerarmos a ação $a_{1}$ quando $\operatorname{Pr}\left[R_{b}\right]>0.5$.

\section{Modelos para o Índice de Produção da Industria Geral.}

Nesta Seção, são apresentados os resultados de diversos modelos aplicados ao índice base fixa da produção da indristria geral permitindo a comparação do desempenho destes modelos quanto a sua capacidade preditiva. Os modelos considerados são: modelo dinâmico Bayesiano básico (MDB) e variantes, modelo estrutural clássico (MEC) e um modelo de função de transferência (MFT). 
A economia brasileira atravessou, nos últimos vinte anos, dois períodos marcadamente diferentes. A década de setenta, de crescimento e estabilidade, e a de oitenta, de instabilidade com períodos de crescimento seguidos de outros de estagnação. Por isto, os modelos terão seus desempenhos avaliados com base nos períodos: 70 a 90 e 80 a 90 .

\subsection{Características dos Modelos e Critérios de Com- paração.}

$\mathrm{O} \mathrm{MDB}$, além da sua versão básica, pode conter uma componente de flutuação cíclica e outra que incorpore o número de dias trabalhados como regressor. Do ponto de vista conceitual, nada garante a existência do ciclo e quanto ao efeito do número de dias, vale lembrar que as indústrias de processo funcionam continuamente e, nas demais, a intensidade do trabalho pode flutuar ao longo dos meses.

Para comparar estas alternativas serão feitas medidas de diagnóstico, que incluem o logarítmo da verossimilhança preditiva (LVP), o Theil-U (TU) e projeções a $h$ passos dentro da amostra para medir a capacidade preditiva através do desvio absoluto médio (DAM).

A análise da conjuntura econômica está, primariamente, interessada na previsão de curto prazo. Assim o DAM avalia a capacidade preditiva do modelo repetindo a previsão fora da amostra para sucessivos instantes de tempo, constituindo-se, portanto, numa medida adequada para comparar modelos alternativos.

Apesar da característica preditiva dos modelos estruturais, o modelo que apresenta o menor erro de previsão a 1 período não é necessariamente o melhor para previsões a maiores prazos. Isto contrasta com a análise de conjuntura que se interessa por aquilo que vai ocorrer nos próximos meses ou trimestres e não no próximo mês, tornando ambíguo o critério de seleção dos modelos.

\section{Comparações no Período 70-90.}

$\mathrm{O} \mathrm{MDB}$, além dos blocos de tendência linear e sazonalidade, incluirá, em alguns casos, o regressor dias úteis e a componente autorregressiva de segunda ordem para representar eventuais ciclos. $\mathrm{O}$ fator de desconto da tendência será, no modelo básico, fixado em .90. 
Será testado, também, o funcionamento do monitoramento Bayesiano. das modificaçōes estruturais ocorridas na série.

O modelo MFT, originalmente identificado utilizando-se dados da década de oitenta, é descrito pela equação abaixo onde $D_{t}$ representa o número de dias úteis.

$$
(1-B)\left(1-B^{12}\right) y_{t}=\beta(1-B)\left(1-B^{12}\right) D_{t}+(1-\alpha B) \epsilon_{t}
$$

Com base em dados mensais do período 1979 a 1988, não se rejeitou a hipótese de que $\alpha$ é igual a um, o que implica nas simplificações descritas em Coelho \& Tenenblat (1989) seguindo Bell (1987). Para fins de comparação, o modelo foi re-estimado para cada um dos dois períodos de análise.

O desempenho do modelo MFT foi comparado com o de três alternativas do modelo MDB. No cálculo das estatísticas foram excluídas as 13 primeiras observações. Uma das alternativas dos MDB utiliza um procedimento automático de intervenção (MDB+MIA) em pontos sinalizados pelo monitoramento Bayesiano e a outra é o modelo básico com uma componente autorregressiva de segunda ordem (MDB+AR) superposta, visando modelar variações cíclicas eventualmente existentes nos dados.

$\mathrm{O}$ efeito do monitoramento Bayesiano é significativo. O TU decresce cerca de $7 \%$ (de .69 para .64), o logarítmo da verossimilhança preditiva é -613 (cresce 68 unidades) e o desvio absoluto médio decresce $10 \%$ (de 3.7 para 3.3). A componente AR tem um efeito menos expressivo. A verossimilhança piora, o TU é praticamente igual ao do modelo básico e o DAM se comporta como no caso anterior. Do ponto de vista da capacidade preditiva, os modelos Bayesianos alternativos se equivalem, superando, porém, expressivamente o MFT como pode ser observado na Tabela 1.

onde: $\mathrm{M}_{h}\left(\mathrm{~T}_{h}\right)$ - significa o DAM a $h$ meses ( $h$ trimestres).

A evolução do desvio médio absoluto para um horizonte de 2 trimestres ao longo do tempo é apresentada na figura 1. Observase que a vantagem do modelo $\mathrm{MDB}$ na década de setenta reduzse gradualmente na década de oitenta, o que é consistente com as características dos modelos. O modelo MFT é simplesmente uma regressão linear envolvendo as diferenças sazonais das variáveis dias 
Tabela 1.

Comparação dos Modelos para Indústria Geral — 70/90

\begin{tabular}{c|cc|ccccc}
\hline & LVP & T-U & M1 & M3 & M6 & T1 & T2 \\
\hline MFT & & & 3.6 & 5.7 & 7.8 & 7.4 & 8.9 \\
MDB & -681 & .69 & 3.7 & 4.6 & 5.5 & 3.9 & 4.9 \\
MDB \& MIA & -613 & .64 & 3.3 & 4.6 & 5.9 & 3.6 & 5.2 \\
MDB \& AR & -43 & .68 & 3.3 & 5.2 & 6.5 & 4.2 & 5.8 \\
\hline
\end{tabular}

uiteis e índice da produção industrial, não apresentando tendência, quer determinística quer estocástica. Esta característica o prejudica na década de setenta e o favorece num período instável como os anos oitenta.

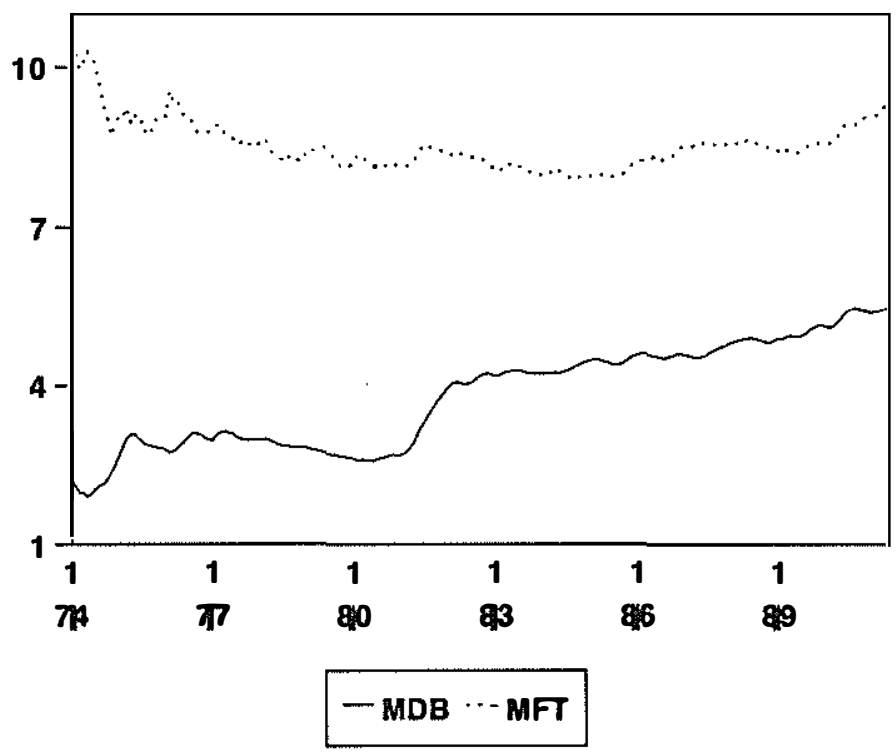

Figura 1.

DAM - horizonte 2 trimestres

MFT $\times$ MDB 
O funcionamento do monitoramento é exibido na figura 2 onde são apresentadas a tendência da série suavizada e seu intervalo de confiança. Neste exercício, os momentos de mudança estrutural, indicados pelo monitoramento, coincidem com instantes de ocorrência de reconhecidas mudanças estruturais na economia.

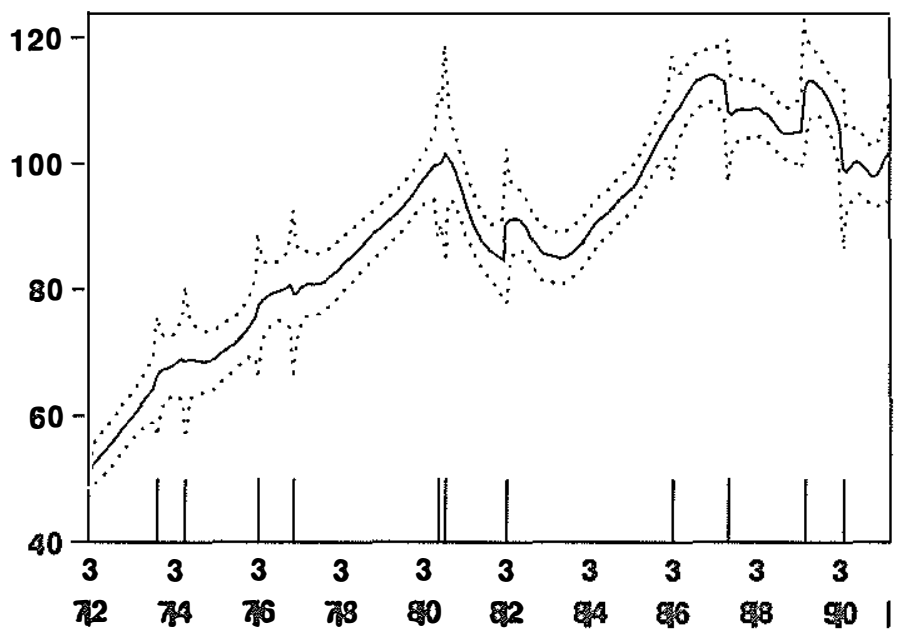

INTERV - Tendencia $\cdots$ Tend $-2^{*}$ DP $\cdots$ Tend $+2^{*}$ DP

\section{Figura 2. \\ PROD. INDUSTRIAL GERAL \\ Tendência Suavizada e Intervenção}

A componente AR(2) do modelo MDB só apresentou raízes complexas conjugadas na década de setenta. Curiosamente, o termo autoregressivo do modelo MDB tem, como se observa na figura 3, uma trajetória semelhante ao da componente cíclica do modelo MEC. Esta semelhança, possivelmente, é consequência do termo estocástico da componente $\mathrm{AR}(2)$ neste último. De qualquer forma, este termo reduziu a capacidade preditiva do modelo, não sendo portanto indicado o seu uso. 


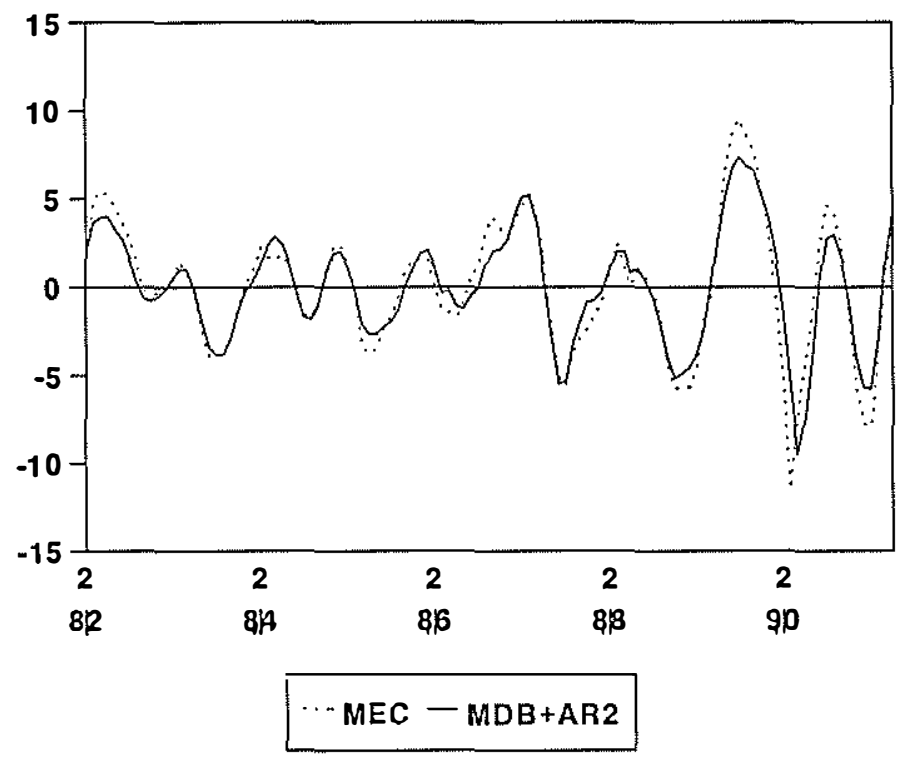

Figura 3.

COMPARAÇĀO CICLO

$\mathrm{MDB} \times \mathrm{MEC}$

Comparação no Periodo 80-90.

$\mathrm{Na}$ década de oitenta utilizou-se, também, o modelo $\mathrm{MEC}$ e versões do modelo $\mathrm{MDB}$ que dão conta da maior instabilidade da série no período através da ampliação da incerteza indicada por menores fatores de desconto para o bloco da tendência (0.85), o que corresponde a redução da 'meia vida' deste bloco para 4 meses.

A tabela 2 trata específicamente do período de maior instabilidade econômica (janeiro de 1980 a maio de 1991). Nela são feitas comparações do modelo Bayesiano com diferentes fatores de desconto, do MEC estimado com dois conjuntos de hiperparâmetros e com o MFT. O logaritmo do fator de Bayes da comparação dos modelos com desconto de .9 e .8 é 17 , o que indica a preferência pelo segundo. Isto caracteriza a volatilidade dos dados neste período. O modelo MEC apresentou um comportamento preditivo a um passo melhor que os modelos $\mathrm{MDB}$ embora não difiram quanto à capacidade preditiva 
para horizontes maiores. Ressalta-se que o comportamento do MFT é ligeiramente (6.3 para 6.5 ou seja $3 \%$ ) melhor que os demais. Isto se deve, possivelmente, ao comportamento dos dados nesta década caracterizado pela instabilidade e a ausência de crescimento. Ainda assim este modelo não é adequado para se prever o agregado de um e dois trimestres, quando a variação relativa é de $40 \%$ (9.9 para 6.0). $\mathrm{O}$ modelo MEC, escolhendo de forma ótima as variâncias de evolução dos parâmetros, 'super-ajusta' os dados como é sugerido pela não melhoria da capacidade preditiva. Cálculos aproximados sugerem que o fator de desconto associado à componente de nível é da ordem de 0.68 no MEC, o que corresponde a uma meia vida de 2 meses.

Tabela 2.

Comparação de Modelos para a Indústria Geral Período 80-90

\begin{tabular}{c|cc|ccccc}
\hline & LVP & T-U & M1 & M3 & M6 & T1 & T2 \\
\hline MFT & & & 3.1 & 5.2 & 6.3 & 7.6 & 9.9 \\
MEC-1 & -346 & .58 & 3.2 & 5.3 & 6.6 & 4.3 & 6.0 \\
MEC-2 & -349 & .56 & 3.3 & 5.2 & 6.5 & 4.2 & 6.0 \\
MDB-D & -393 & .56 & 4.9 & 5.8 & 6.8 & 4.9 & 6.2 \\
MDB-D(.8) & -376 & .49 & 4.0 & 5.5 & 6.7 & 4.6 & 6.4 \\
\hline
\end{tabular}

$\mathrm{Na}$ tabela 3 diferentes versões do modelo MDB são comparadas. Coerente com o cenário de instabilidade e estı̇nação da década de oitenta o melhor modelo é o estático Bayesiano (MEB), que supõe a ausência da tendência de crescimento e mudanças estruturais. Para facilitar a identificação dos momentos de reversão aplicou-se o MDB à média móvel $(M D B+M M)$ da série. Observa-se que se por um lado o processo de média móvel elimina a instabilidade da série, por outro piora a capacidade preditiva devido, possivelmente, à perda de informação indicada pela função de previsão.

\subsection{Determinação de Pontos de Reversão Cíclica.}

O procedimento de indicação de reversão foi aplicado sobre as 
Tabela 3.

Comparação de MDB para a Indústrial Geral Periodo 80-90

\begin{tabular}{ccccccc}
\hline & \multicolumn{3}{c}{ S/Mon. } & \multicolumn{3}{c}{ C/Mon. } \\
\hline & M1 & M6 & T2 & M1 & M6 & T2 \\
\hline MDB & 5.0 & 6.9 & 6.1 & 4.8 & 7.6 & 6.6 \\
MDB-D & 4.9 & 6.8 & 6.2 & 4.8 & 7.3 & 6.8 \\
MDB-D(.85) & 4.3 & 6.5 & 6.0 & 4.3 & 7.3 & 6.9 \\
MEB-D & 6.1 & 7.1 & 6.7 & 5.0 & 6.3 & 5.9 \\
MDB+MM & 7.6 & 15.8 & 14.2 & 7.2 & 15.9 & 14.5 \\
\hline
\end{tabular}

componentes estimadas da série da produção da indústria geral. A probabilidade de reversão foi calculada de forma aproximada por:

$$
\operatorname{Pr}\left[R_{b} \mid D_{t}\right]= \begin{cases}\operatorname{Pr}\left[\mu_{t+1}<m_{t} \mid D_{t}\right], & \text { se } M_{t-1}<M_{t} \\ 0 & \text { em caso contrário }\end{cases}
$$

onde $M_{t}$ é a média móvel da série e $m_{t}$ a média da distribuição a posteriori.

$\mathrm{Na}$ figura 4, as linhas menores representam reversão para cima e as longas para baixo. Os resultados demonstram a capacidade do método em indicar as reversōes adequadamente. Este procedimento apontou corretamente 8 das reversões ocorridas. É necessário destacar que estas decisões são baseadas na distribuição a posteriori em cad a instante de tempo e, em consequência, obtidas sequencialmente.

\section{Projeçōes das Séries Econômicas.}

A conjuntura econômica é descrita por variáveis nominais e reais. No Brasil a extrema volatilidade dos preços enfraquece, no caso de séries nominais, o conceito de tendência e esconde uma eventual sazonalidade. Portanto este tipo de instrumental não é recomendável para séries nominais. As séries reais selecionadas podem ser agrupadas nas que:

- descrevem o produto da indústria, indicador geral e desagregado por uso; 


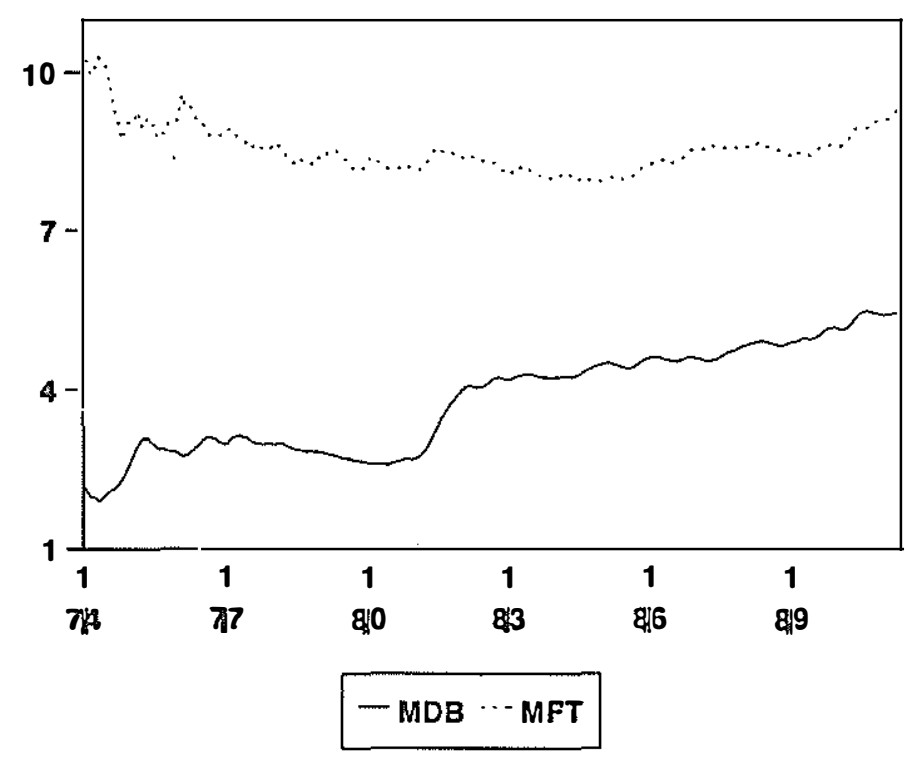

Figura 4.

Indicador de Reversão - Indústria Geral

- determinam o produto interno bruto segundo a metodologia do PIB trimestral que inclui indicadores de atividade dos setores mais importantes da economia;

- contabilizam o desempenho da balança comercial;

- indicam o nível de consumo real em São Paulo medindo o estado de "aquecimento" da economia em diguns segmentos mais importantes.

As séries que têm grande alteração do seu nível deixam em aberto a possibilidade da componente sazonal ser proporcional ao seu nível o que exigiria o uso de modelo multiplicativo. De outro lado séries que não tenham marcada trajetória de crescimento - e são muitas na década de oitenta no Brasil - poderiam admitir modelos de primeira ordem. Para cada série foram testadas diferentes opções do modelo linear de crescimento com componentes sazonais aditivas. Os fatores de desconto utilizados foram os recomendados na literatura e servem para controlar a adaptabilidade dos parâmetros. 


\subsection{Indices de Produção da Indústria.}

O IBGE apura, a partir de uma amostra do tipo painel, um índice de quantidade do produto da indústria. Este índice pela sua abrangência e cuidado metodológico é amplamente utilizado como indicador nacional do nível de atividade. Além deste são apurados índices do produto industrial agregado por gênero de indústria e por uso do bem produzido. Foram selecionados para a previsão o índice da indústria geral e os agregados por uso. Estes últimos, devido a sua definição, não são uma desagregação do índice geral.

O procedimento adotado consistiu em utilizar modelos para cada uma das séries isoladamente, exclusive uma - a de bens de consumo intermediário - que será calculada a partir das previsões das demais, de forma a garantir a coerência entre as previsōes dos índices. Um modelo relacionando o índice de bens de consumo intermediários com os valores contemporâneos e defasados dos demais índices e, ainda, da própria série em estudo foi ajustado aos dados.

Os modelos utilizados nos índices de produção de bens de consumo durável e não durável incluíram ou não dias úteis como variável explicativa e o uso de monitoramento dos erros de previsão. Este expediente só apresentou resultados marginalmente significativos para a série dos bens de consumo duráveis.

Tabela 4.

Comparação de Modelos para índices de Bens de Consumo Desvio Absoluto Médio

\begin{tabular}{crrrrrr}
\hline & \multicolumn{3}{c}{ S/ Mon. } & \multicolumn{3}{c}{ C/Mon. } \\
\hline & M1 & M6 & T2 & M1 & M6 & T2 \\
\hline Durável & & & & & & \\
MDB & 9.7 & 11.0 & 9.3 & 9.2 & 10.5 & 9.0 \\
MDB- D & 9.2 & 10.6 & 9.9 & 8.5 & 11.1 & 10.2 \\
\hline Não Durável & & & & & & \\
MDB & 5.3 & 7.6 & 6.8 & 5.1 & 8.2 & 7.3 \\
MDB-D & 4.9 & 7.4 & 7.1 & 4.5 & 8.5 & 8.4 \\
\hline
\end{tabular}


A previsão do índice de produção da indústria de transformação pode ser derivado dos índices da indústria geral e da extrativa mineral pois ponderadores fixos os relacionam entre si. Como já foi comentado, os índices agregados por uso não estão relacionados de forma determinística com o índice geral da produção industrial. O procedimento descrito, que realiza projeções compatíveis para as duas desagregaçōes - por uso e gênero - resulta, num erro absoluto médio para previsões da série de bens de consumo intermediário, realizadas com 3 períodos de antecipação, de $6.4 \%$.

A tabela abaixo apresenta o resultado para os índice de produção das indústrias extrativa mineral e de bens de capital. $\mathrm{O}$ caso da indústria extrativa mineral apresenta tendência e sazonalidade marcantes, cabendo então cogitar do efeito da sazonalidade proporcional e, também, testar a contribuição dos dias úteis. A inclusão dos dias úteis e o uso de modelos sazonais multiplicativos não apresentam vantagens significativas, enquanto que o monitoramento melhora a capacidade preditiva.

Tabela 5 .

Modelos para Extrativa Mineral e Bens de Capital

Desvio Absoluto Médio

\begin{tabular}{crrrrrr}
\hline & \multicolumn{3}{c}{ S/ Mon. } & \multicolumn{3}{c}{ C/Mon. } \\
\hline & M1 & M6 & T2 & M1 & M6 & T2 \\
\hline Extrativa Mineral & & & & & & \\
MDB & 6.3 & 10.6 & 9.3 & 5.0 & 8.8 & 7.3 \\
MDB- D & 6.5 & 10.6 & 9.5 & 5.1 & 8.8 & 7.9 \\
MDB-M & 4.3 & 9.8 & 8.0 & 5.1 & 10.0 & 7.7 \\
\hline Bens de Capital & & & & & & \\
MDB & 6.6 & 9.7 & 8.6 & 6.1 & 9.5 & 8.4 \\
MDB-D & 6.7 & 9.6 & 9.0 & 6.1 & 9.5 & 8.7 \\
MDB+AR & 6.2 & 9.9 & 8.5 & & & \\
\hline
\end{tabular}

No caso do índice de produção de bens de capital testou-se modelos com dias úteis e com a inclusão de uma componente autore- 
gressiva. Ambos não apresentam resultados significativos. O monitoramento, por outro lado, melhora marginalmente os resultados.

\subsection{Componentes do PIB Real Trimestral.}

O PIB trimestral é um índice de Laspeyres encadeado que compõe índices de produção (de oferta) dos principais grupos de atividade da economia. As séries apresentadas nas tabelas 6 e 7 são as principais componentes deste indicador. As componentes relativas aos índices de produção da indústria de transformação e extrativa mineral já foram consideradas anteriormente. Os índices de produção do setor agrícola serão omitidos pois não são produzidos mensalmente, mas construídos a partir de medidas de prrdução anual dos principais produtos e do padrão sazonal das correspondentes safras.

Tabela 6.

Comparaçāo de Modelos para os índices do Produto Interno Bruto

\begin{tabular}{ccccccc}
\hline & \multicolumn{3}{c}{ S/ Mon. } & \multicolumn{3}{c}{ C/Mon. } \\
\hline & M1 & M6 & T2 & M1 & M6 & T2 \\
\hline Energia El. & & & & & & \\
MDB & 3.5 & 4.7 & 3.8 & 3.4 & 5.0 & 4.1 \\
MDB-M & 3.6 & 4.6 & 3.8 & 4.1 & 6.4 & 5.1 \\
\hline Óleo Diesel & & & & & & \\
MDB & 6.8 & 7.7 & 5.8 & 7.1 & 8.9 & 6.9 \\
MDB-M & 7.8 & 8.3 & 6.1 & 8.7 & 12.9 & 9.2 \\
\hline C. Civil & & & & & & \\
MDB & 6.0 & 10.3 & 9.4 & 5.0 & 10.1 & 9.2 \\
\hline Pecuária & & & & & & \\
MDB & 5.5 & 7.7 & 7.1 & 5.2 & 8.3 & 7.4 \\
MDB-AR & 5.6 & 10.3 & 8.8 & & & \\
\hline
\end{tabular}

O consumo de energia elétrica mede a produção dos serviços de utilidade pública. Apresenta uma tendência e padrão sazonal bem definido. Avaliou-se o efeito de componentes sazonais multiplicativas, 
que não resultou em melhoria e, também, a inclusão do monitoramento, que não foi vantajosa. O consumo de óleo diesel, proxy para a produção do setor de transportes, tem uma trajetória semelhante à da série anterior. Os modelos multiplicativos e o monitoramento não foram satisfatórios.

Tabela 7.

Comparação de Modelos para os indices de Empregos Setoriais

\begin{tabular}{ccccccc}
\hline & \multicolumn{3}{c}{ S/ Mon. } & \multicolumn{3}{c}{ C/Mon. } \\
\hline & M1 & M6 & T2 & M1 & M6 & T2 \\
\hline Industrial & 2.0 & 4.4 & 3.9 & 1.2 & 3.4 & 3.0 \\
Financeiro & 1.7 & 4.4 & 3.9 & 0.8 & 3.3 & 2.8 \\
Outros Serviços & 1.8 & 3.3 & 2.9 & 1.3 & 3.0 & 2.7 \\
\hline
\end{tabular}

O índice de produção de insumos da construção civil é uma proxy do produto dos serviços deste setor. O uso do monitoramento apresenta melhoras apenas marginais. A produção pecuária é um índice composto por diversos componentes, das quais a produção de carne bovina é a que tem o maior peso. A literatura menciona a existência de um ciclo de cerca de 4 anos para esta produção, que está relacionado com o período de maturação dos animais para o abate. Para captar este ciclo foi incluída a componente cíclica. De fato o monitoramento e a inclusão desta componente não melhoraram as previsões, indicando a já habitual "sobreparametrização".

A atividade dos setores de serviços não comércio e das instituições financeiras foi representada por séries de emprego setoriais - na hipóteses de produtividade constante da mão de obra. Por semelhança de natureza foi incluída também a série do emprego na indústria. Todas as séries se caracterizam por um comportamento menos instável e sem componentes sazonais. Em todos os casos o monitoramento apresentou um desempenho significativo, melhorando em até $40 \%$ a capacidade preditiva dos modelos. 


\subsection{Componentes da Balança Comercial.}

A desagregação habitual, que foi adotada, das componentes da balança comercial permite um acompanhamento mais atualizado das informações, pois é a publicada com agilidade pelo Departamento de Comércio Exterior, além de conter algum conteúdo econômico. A pauta de exportação é separada em produtos básicos, manufaturados e semi-manufaturados, grupos que têm apresentado um comportamento específico e especialmente os básicos que estão mais sujeitos às condições do mercado de commodities. De outro lado as importaçōes de petróleo por sua natureza administrada torna mais previsivel o comportamento do agregado de importações que a exclui. Vale relembrar o objetivo de ajustamento de um modelo descritivo que permita extrapolar a tendência da série, o que exclui o uso da taxa de câmbio que seria a variável chave para explicar boa parte do comportamento destas séries. A exportação de manufaturados e a importação exclusive petróleo tem um padrão de crescimento, ainda que instável, justificando a tentativa de uso de modelos multiplicativos.

Tabela 8.

Comparação de Modelos para os índices da Balança

Comercial - Desvio Absoluto Médio

\begin{tabular}{ccccccc}
\hline & \multicolumn{3}{c}{ S/ Mon. } & \multicolumn{3}{c}{ C/Mon. } \\
\hline & M1 & M6 & T2 & M1 & M6 & T2 \\
\hline Exportação & & & & & & \\
Manufaturados & 21.9 & 32.7 & 27.3 & 20.3 & 33.5 & 28.3 \\
Semi-Manufat. & 25.3 & 36.1 & 30.7 & 25.0 & 40.5 & 32.6 \\
MDB-M & 26.2 & 37.9 & 31.0 & 25.9 & 40.6 & 30.0 \\
Básicos & 12.7 & 17.3 & 15.4 & 12.3 & 19.9 & 17.8 \\
\hline Importação & & & & & & \\
Exc. Petróleo & 9.5 & 14.3 & 12.0 & 9.0 & 14.0 & 11.8 \\
MDB-M & 9.3 & 14.1 & 11.4 & 9.8 & 18.0 & 13.6 \\
\hline
\end{tabular}


Os resultados apresentados na tabela 8 mostram que o uso de modelos multiplicativos ou de monitoramento não melhora o péssimo desempenho preditivo dos modelos. Chama a atenção que o desvio médio absoluto é três vezes maior que os casos anteriormente expostos, tornando, praticamente, inútil o uso destes modelos para extrapolação. Vale dizer que este resultado retrata a instabilidade intrínsica das séries, tornando igualmente imprecisos quaisquer procedimentos de extrapolação.

\subsection{Indicadores de Consumo.}

Os indicadores de consumo são agregados nominais coletados pela Federação de Comércio de São Paulo e, posteriormente, deflacionados por algum índice de preços. A coleta realizada por uma instituição não especializada e o deflacionamento de valores nominais deixa alguma dúvida quanto a qualidade das séries. Além disso cobrem apenas a cidade de São Paulo reduzindo a sua abrangência. Apesar destes pontos negativos são as únicas medidas do lado da demanda.

Tabela 9.

Comparação de Modelos para os índices de Consumo em São Paulo - Desvio Absoluto Médio

\begin{tabular}{crrrrrr}
\hline & \multicolumn{3}{c}{ S/ Mon. } & \multicolumn{3}{c}{ C/Mon. } \\
\hline & M1 & M6 & T2 & M1 & M6 & T2 \\
\hline Geral & 7.1 & 11.0 & 9.7 & 6.2 & 10.9 & 9.3 \\
\hline Bens de Consumo & & & & & & \\
Não Durável & 10.5 & 18.6 & 16.6 & 7.8 & 16.1 & 13.8 \\
Semi-Durável & 10.3 & 13.7 & 12.2 & 9.8 & 16.1 & 14.0 \\
Durável & 8.2 & 11.4 & 10.3 & 6.7 & 11.9 & 10.2 \\
\hline Bens de Investimento & & & & & & \\
Veículos & 8.9 & 13.0 & 11.5 & 8.6 & 13.4 & 11.7 \\
Mat. Construção & 7.0 & 10.5 & 9.5 & 6.3 & 11.1 & 9.9 \\
\hline
\end{tabular}


Os resultados apresentados na tabela 9 mostram que o mesmo padrão, o efeito do monitoramento é marginal, melhorando a capacidade descritiva, mas melhorando muito pouco e em alguns casos piorando a capacidade preditiva. A qualidade preditiva medida pelo desvio absoluto médio do erro de previsão no segundo trimestre é da mesma magnitude - cerca de $10 \%$ - dos casos anteriores, exceto para os componentes da balança comercial.

\section{Conclusão.}

Neste artigo comparou-se modelos alternativos aplicados à série do produto da indústria geral. Os resultados sugerem que os modelos estruturais têm capacidade preditiva tão boa ou melhor do que o não estrutural tomado como exemplo e que a abordagem Bayesiana também apresenta resultados equivalentes aos da clássica. Conceitualmente os modelos estruturais têm a característica de descreverem qualitativamente os atributos das séries - tendência, sazonalidade e a abordagem Bayesiana dos modelos estruturais é, adicionalmente, mais simples no uso, além de dispor dos recursos de monitoramento e intervenção - que aumentam a capacidade de descrever os fatos econômicos.

O procedimento de intervenção utilizado se limitou às indicações do monitoramento, apesar de potencialmente ser capaz de incorporar a opinião dos especialistas. Isto é possibilitado pelo fato dos parâmetros do modelo terem interpretação econômica, e serem conceitualmente variáveis aleatórias cuja distribuição de probabilidade pode ser imputada subjetivamente. Vale dizer que, apesar de não terem sido apresentados o gráfico dos resultados, o monitoramento indicou corretamente, para a maioria das séries, os seus momentos de mudança de inclinação.

O desempenho do modelo nas séries foi heterogêneo - erros médios absolutos variando de $2 \%$ a $12 \%$ - indicando a diversidade das características do conjunto de séries analisado. Particularmente as séries relativas ao comércio exterior foram as que apresentaram o pior desempenho. As características probabilísticas e de flexibilidade dos modelos estruturais Bayesianos sugerem que métodos alternativos que se limitam a extrapolar a trajetória da série terão 
também um desempenho igualmente ruim no conjunto de séries mais volá teis.

(Submetido em abril 1993. Revisado em novembro de 1993)

\section{Referências}

Bell, W 1987. "A note on Overdifferencing and the Equivalence of Seasonal Time Series with Monthly means and Models with $(0,1,1)$ Seasonal parts when $(\mathrm{q}=1)$." J.of Bussiness and Econ. Statistics, 5:3.

Coelho, C.H.M \& Tenenblat, M 1992. "Forecasting with Trading Day Variation, a Seasonal Common Factor and Variance Change." International Journal of Forecasting, 8:61-68.

Gamerman, D. \& Migon, H. S. 1993. "Hierarchical Dynamic Models." To Appear in JRSS-B, 55.

Harrison, P. J \& Stevens, C. F. 1976. "Bayesian Forecasting." $J R S S-B, 38: 205-247$.

Harvey, A.C. 1981. Time Series Models. Oxford: Philip Allan.

Harvey A.C. \& Outros 1989. Stamp - Structural Time Series Package.

Migon, H. S. \& Brasil, G. H. 1986. Modelos de Previsão para Séries de Produção e Preços: métodos Bayesianos e Box-Jenkins IPEA-RJ.

Migon, H. S. \& Gamerman, D. 1993. "Generalized Exponential Growth Models: a Bayesian Approach." To appear in J. of Forecasting.

Pereira, P.V. \& Harvey, A.C. 1988. "Trend, Seasonality and Seasonal Adjustment." TDI $\mathrm{n}^{2}$ 154, IPEA.

West, M.\& Harrison P. J. 1989. Bayesian Forecasting and Dynamic Models. Springer Verlag.

Zellner A., Hong C. \& Gulati G.H. 1987. "Turning Points in Economic Time Series, Loss Strutures and Bayesian Forecasting." Graduate School of Business University of Chicago, Discussion Paper. 


\section{ERRATA}

Ao artigo "Componentes principais estocásticamente restritos" do prof. Hugo Boff, publicado na Revista de Econometria - Ano XIII, número-1. Abril de 1993:

"A Seção (4) referida no Resumo e no Abstract é, na realidade, Seção 5. À pg. 24, em nota 2, menciona outro texto nosso, "Variáveis padronizadas...", o qual contém algumas incorreções que, de fato, não o habilitam a referir sobre o tema. À pag. 29 , reenvia-se o leitor à seção 4, e não 3.4 (inexistente)." 